\title{
Specialists, GDPs and patients, and their attitudes to biopsy
}

\author{
Attitudes to biopsy procedures in general dental practice
}

\section{N. Diamanti, A. J. Duxbury, S. Ariyaratnam and T. V. Macfarlane Br Dent J 2002; 192: 588-592}

\section{Aim}

To investigate biopsy procedures in general dental practice.

\section{Objectives}

To assess the views and attitudes of: specialists on the dental specialist surgical registers; dentists in general practice (GDPs) and patients undergoing biopsy procedures.

\section{Method}

Questionnaires were sent to 98 oral and maxillofacial surgeons and surgical dentists, 335 general dental practitioners and 220 patients attending the Oral Medicine Clinic at the Dental Hospital, Manchester. Participation rates were 68 (74\%), 227 (72\%), and 158 (76\%) respectively.

\section{Results}

Specialists: 47 (70\%) would discourage dental practitioners undertaking biopsies. Concerns were a lack of skills and delays in referral; 20 (30\%) considered GDPs should be able to perform simple biopsies for benign lesions. Dentists: 33 (15\%) reported they had performed oral biopsies in the last two years; 136 (60\%) felt they should be competent to biopsy benign lesions. Their main concerns were lack of practical skills and the risk of diagnostic error. Patients: 112 (65\%) worried about their biopsy result, 67 (39\%) would feel anxious if their dentist did the biopsy, although $40(23 \%)$ were anxious when biopsied in the oral medicine clinic.

\section{Conclusions}

Both specialists on the dental surgical registers and GDPs feel there is a need for further training in biopsy technique for GDPs and better advertised and accessible pathology support. The current fee for biopsies may need upward revision. A main concern of patients is fear of an adverse biopsy report. Whilst patients are satisfied with specialist management any concerns were an insufficient reason for biopsy of a benign lesion not being undertaken in general practice.

\section{IN BRIEF}

Shows the attitudes of specialists on the Dental Surgical Register to dental practitioners undertaking biopsies.

1 Indicates the views of dental practitioners on biopsy in practice and a need for more postgraduate training.

Suggests that current fees for biopsies undertaken in general practice are inadequate.

Reveals patients' worries about biopsy procedures.

\section{COMMENT}

This is a timely paper on attitudes to oral biopsy procedures and its message benefits from publication in a general dental journal. In this retrospective study the authors sought the views of oral and maxillofacial surgeons, specialists in surgical dentistry and general dental practitioners, as well as patients having oral biopsies. Specialists on the GDC oral medicine list do not appear to have been consulted. This questionnaire-based study had excellent rates of return (maxillofacial surgeons/surgical dentists 74\%, general dental practitioners $72 \%$ and biopsy patients $76 \%$ ).

Surgical specialists consider that overall, dentists should refer lesions for biopsy, though those that felt dentists could perform biopsies emphasised biopsy procedures for benign lumps and bumps rather than, presumably, white patches, red patches and ulcers. Patients were anxious whoever was performing the biopsy and unsurprisingly expressed a preference for technical competence in the operator. Patients were less anxious when biopsied by a specialist. Only $15 \%$ of general dental practitioners reported performing biopsy procedures within the two years prior to the questionnaire. No information is given as to how recently this group qualified, nor previous surgical experience. Reasons for this rather low percentage included having had no training (39\%), concerns relating to diagnostic error and lack of awareness of materials and transport for the specimens obtained. The rather low NHS fee payable for oral biopsy was also suggested as a disincentive for biopsy in general practice. Dentists should be aware of the availability of specimen pots and pathology forms from local hospital oral pathology departments.

It is to be hoped that papers such as this will raise the awareness of general dental practitioners as to the possibility of performing simple, excisional biopsies of benign lesions in practice as a service to patients and to widen the skills used in the practice setting.

There is always a pool of general dental practitioners who have had surgical experience via oral surgery or oral medicine senior house officer posts. This pool will increase in size due to a series of factors: an increased emphasis on general professional training (GPT) schemes for newly qualified dentists; the development of surgical dentistry outside of hospitals; and the likely increase in demand for relevant CPD courses to fulfil GDC requirements (which should include courses in biopsy techniques for dental practitioners). In addition, increasing the number of dentists assuming the role of oral physician will help in educating patients that dentists are more than tooth technicians. The above, accompanied by relevant research such as that found in this paper, can only serve to promote positive attitudes to biopsy procedures in dental practice.

Dr Philip Atkin,

Specialist Registrar/Honorary Clinical Lecturer in Oral Medicine, Royal London Hospital 